\title{
Vitamin E Has Reduced Affinity for a Polyunsaturated Phospholipid: An Umbrella Sampling MD Simulations Study
}

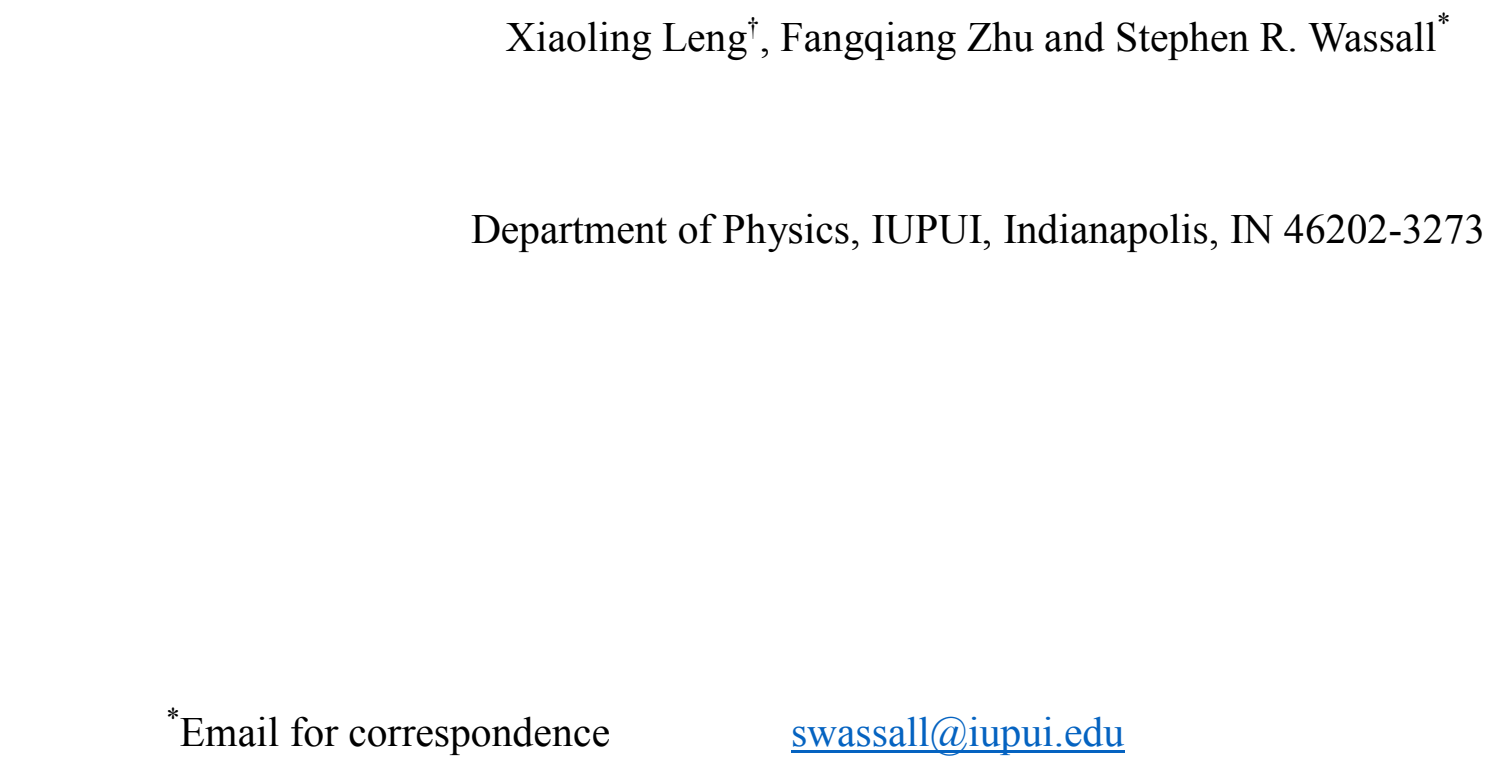

${ }^{\dagger}$ Current address Institute of Molecular Biophysics, Florida State University, Tallahassee, FL 32306-4380

This is the author's manuscript of the article published in final edited form as: 


\begin{abstract}
Vitamin $\mathrm{E}$ is an essential micronutrient. The primary function of this lipid soluble antioxidant is to protect membrane phospholipids from oxidation. Whether vitamin $\mathrm{E}$ preferentially interacts with polyunsaturated phospholipids to optimize protection of the lipid species most vulnerable to oxidative attack has been an unanswered question for a long time. In this work we compared the binding of $\alpha$-tocopherol ( $\alpha$ toc), the form of vitamin E retained by the human body, in bilayers composed of polyunsaturated 1-stearoyl-2docosahexaenoylphosphatidylcholine (SDPC, 18:0-22:6PC) and, as a control, monounsaturated 1-stearoyl-2-oleoylphosphatidylcholine (SOPC, 18:0-18:1PC) by umbrella sampling molecular dynamics (USMD) simulations. From the potential of mean force (PMF) as a function depth within the bilayer, we find that the binding energy of atoc is less in $\operatorname{SDPC}\left(\Delta G_{\text {bind }}=16.7 \pm 0.3\right.$ $\mathrm{kcal} / \mathrm{mol})$ than in $\operatorname{SOPC}\left(\Delta G_{\text {bind }}=18.3 \pm 0.4 \mathrm{kcal} / \mathrm{mol}\right)$. The lower value in SDPC is ascribed to the high disorder of polyunsaturated fatty acids (PUFA) that produces a less tightly packed arrangement. Deformation of the bilayer is observed during desorption, indicating that phosphatidylcholine (PC)-PC, as well as atoc-PC, interactions contribute to the binding energy. Our results do not support the proposal that vitamin E interacts more favorably with polyunsaturated phospholipids.
\end{abstract}




\section{INTRODUCTION}

Vitamin E stands for the name of a group of naturally occurring, biologically active tocol (tocopherol and tocotrienol) compounds. ${ }^{1,2} \alpha$-Tocopherol ( $\alpha$ toc) is the form that is retained in the human body. This lipid-soluble antioxidant is an essential micronutrient. Neurological problems are among the symptoms of its deficiency in the diet. ${ }^{3}$ There is general agreement that the primary function for $\alpha$ toc is to protect polyunsaturated lipids that are extremely susceptible to attack from free radicals in membranes. ${ }^{2,4-6}$ The growing consumption of fish oil supplements rich in omega-3 polyunsaturated fatty acids (n-3 PUFA) that are taken up into membrane phospholipids underscores the importance of this role. ${ }^{7-10}$ Although the chemistry by which atoc inhibits the peroxidation of lipid chains is known, ${ }^{11-12}$ an open question is whether preferential interaction with polyunsaturated lipids exists. ${ }^{13-15}$

The atoc molecule is composed of a chromanol head group with a hydroxyl group at one end and a phytyl chain at the other end (Figure 1). It usually resides in a membrane with the hydroxyl group near the aqueous interface while the phytyl chain extends towards the center. ${ }^{16-18}$ In this arrangement the sacrificial hydroxyl group traps lipid peroxyl radicals when they rise up to the surface of the membrane and subsequently is recycled by a water-soluble reducing agent such as ascorbate. ${ }^{19}$ The general features of the location and orientation of atoc in phospholipid bilayers are reproduced in the few atomistic molecular dynamics (MD) simulations that have been published to date. ${ }^{20-22}$ Our simulations demonstrated how efficiently atoc is able to fulfill its antioxidant role in a polyunsaturated phospholipid bilayer. ${ }^{22}$ They revealed that high disorder enhances the probability that a polyunsaturated chain comes into close proximity with the hydroxyl group on a chromanol group that resides at the surface of the bilayer. Additionally, we observed flip-flop for atoc is fast, which suggests the hydroxyl group can also easily penetrate 
into the interior of a polyunsaturated membrane to intercept lipid peroxyl radicals.

Here we follow up on our earlier simulations with an umbrella sampling molecular dynamics (USMD) simulation study that further compares the molecular organization of atoc in 1-stearoyl-2-docosahexaenoylphosphatidylcholine (SDPC, 18:0-22-6PC) and 1-stearoyl-2oleoylphosphatidylcholine (SOPC, 18:0-18:1PC) bilayers. The molecular structure of SDPC with saturated stearic acid (SA, 18:0) for the sn-1 chain and docosahexaenoic acid (DHA, 22:6) containing 6 double bonds for the $s n-2$ chain is representative of a polyunsaturated phospholipid. ${ }^{23}$ SOPC where the $s n-2$ chain is replaced by oleic acid (OA, 18:1) that has a single double bond serves as a monounsaturated control (Figure 1). From the results, we obtain estimates of the binding energy and rate of flip-flop for atoc and gain insight into the two processes. Does atoc prefer polyunsaturated phospholipids, as has been hypothesized, ${ }^{13,15}$ is a question that we address.

\section{METHODS}

\section{MD simulations}

We prepared two lipid membrane systems: SDPC with atoc and SOPC with atoc. In each case there are in total 80 phosphatidylcholine (PC) lipid molecules and 2 atoc molecules, which are divided equally between the two leaflets. The initial structures of these lipid bilayers were taken from our earlier published simulations containing $20 \mathrm{~mol} \%$ atoc, which have been equilibrated and run for over $200 \mathrm{~ns} .^{22}$ We deleted the extra atoc molecules, leaving two of them - one in each leaflet - to get the bilayer content we needed. In order to ensure enough room to allow an atoc molecule to be fully pulled out of the bilayer into the aqueous phase, the bilayers were hydrated with 8562 water molecules that made a water layer of approximately $45 \AA$ on each 
side. A harmonic constraint was applied to the two atoc molecules to keep them $35 \AA$ apart in the $x y$ plane, which is approximately $1 / 2$ the diagonal length of the bilayer, to minimize interactions between them during the umbrella sampling process. We used the CHARMM C36p force field for all simulations. ${ }^{24}$ Non-bonded (van der Waals and short-range electrostatic) interactions were cut off at $12 \AA$ by a switching function starting at $10 \AA$ and the long-range electrostatic interactions were calculated using the particle-mesh Ewald method with grid spacing $1.0 \AA^{25}$ Periodic boundary conditions were applied to a rectangular box in which the $x$ and $y$ dimensions (in the plane of the membrane) were held equal while the $z$ dimension (in the direction of the membrane normal) was varied independently. The temperature and pressure were kept at $37{ }^{\circ} \mathrm{C}$ and $1 \mathrm{~atm}$, respectively, by the Langevin dynamics (damping coefficient 1/ps) and piston method. $^{26}$

\section{Umbrella sampling}

With USMD simulations we obtained the free energy, known as the potential of mean force (PMF), as a function of atoc's distance from the bilayer center. Then the energy cost of pulling an atoc molecule out from the bilayer, which we call the binding energy, was determined from the corresponding PMF profile.

A brief description of our procedure follows. The distance along the direction $(z)$ of the membrane normal between the center of the bilayer and the center of mass of the rigid chromanol head group on each atoc molecule was chosen to represent the location of atoc - the reaction coordinate. The atoc molecules in both leaflets were restrained symmetrically with respect to the center of bilayer, which maintains the compositional balance of the bilayer and doubles the sampling efficiency. 45 windows were simulated with the value of $z_{i}$ varying from 0 
to $44 \AA$ in increments of $1 \AA$. In each window $i$, the atoc molecules were restrained by a harmonic potential

$$
U_{i}=\frac{1}{2} k\left(z-z_{i}\right)^{2}
$$

with a force constant $k=3.68 \mathrm{kcal} /\left(\mathrm{mol} \cdot \AA^{2}\right)$ that was chosen to optimize the separation between Gaussian distributions in adjacent windows according to an established criterion ${ }^{27}$ at the corresponding $z_{i}$ value. The initial structures in the windows were generated by a series of simulations that pulled the $\alpha$ toc molecules from the equilibrium position to $z=0 \AA$ or $z=44 \AA$ with a speed of $1 \AA$ /ns. The Hamiltonian replica exchange method was applied during umbrella sampling to optimize the process. ${ }^{28}$ Production runs were performed with NAMD $2^{29}$ on the Big Red II super computer at Indiana University. Their duration was $200 \mathrm{~ns}$ in each window, with the last $100 \mathrm{~ns}$ of the trajectories employed to perform the PMF calculation using the weighted histogram analysis method (WHAM) ${ }^{30,31}$ In the WHAM calculation, bin size is set to $0.01 \AA$, the lower and upper boundaries in SDPC are $-1.95 \AA$ and $55.92 \AA$ and in SOPC are $-1.77 \AA$ and $55.74 \AA$. The numerical optimization was done by the BFGS algorithm, with the convergence tolerance set to $2^{-52}$, the minimum relative change that can be represented by a double-precision floating-point number. Errors were estimated following a previously described protocol ${ }^{31}$ in which the statistical errors of the average positions are calculated by the block averaging method. $^{32}$

\section{RESULTS}

\section{Potential of mean force}

Figure 2 shows the PMF $(G(z))$ vs. position relative to the center of the bilayer calculated from our simulations for atoc in SDPC and SOPC. As expected, the shape of the plots resembles 
that reported for cholesterol ${ }^{33,34}$ where, like $\alpha$ toc, the sterol consists of a rigid moiety (tetracyclic ring) with a hydroxyl group at one end and a branched chain at the opposite end. ${ }^{15}$ The variation of $G(z)$ is flat where atoc becomes completely disengaged from the membrane ( $z \geq 35 \AA)$ in the water, and the baseline $(G=0)$ is set there. When atoc is brought into contact with phospholipid, $G(z)$ starts to drop as atoc penetrates more deeply within the membrane until the equilibrium location is reached (where $G(z)=G\left(z_{e q}\right)$ exhibits a minimum). Then there is small increase in $G(z)$ towards the center of the bilayer $(z=0)$ that represents a barrier to movement from one side of the bilayer to the other. As in analogous studies on cholesterol, ${ }^{34}$ we define the binding energy of atoc in the membrane to be $\Delta G_{b i n d}=-G\left(z_{e q}\right)$ and the free energy barrier for the flipflop of atoc between leaflets to be $\Delta G_{f l i p}=G(0)-G\left(z_{e q}\right)$. Table 1 lists the magnitude of these quantities for SOPC and SDPC. Also included in the table are values for the equilibrium depth $z_{e q}$ of atoc in each bilayer (10.1 $\AA$ in SDPC and $11.4 \AA$ in SOPC), which qualitatively agree with estimates made from our previous standard all-atom MD simulations (11.3 $\AA$ in SDPC and $11.8 \AA$ in SOPC). ${ }^{22}$ Exact agreement is not be expected in view of the increase in bilayer order and thickness that accompanies the higher concentration of the vitamin in the earlier work (20 vs. $2.5 \mathrm{~mol} \%)$.

\section{Binding energy}

The binding energy $\Delta G_{\text {bind }}$ evaluated here for atoc by UBSMD simulations in SDPC $(16.7 \mathrm{kcal} / \mathrm{mol})$ is less favorable than in $\operatorname{SOPC}(18.3 \mathrm{kcal} / \mathrm{mol})$ (Table 1). These values are comparable to the few other reports to date of a binding energy from USMD simulations for atoc in PC bilayers, ${ }^{35,36}$ although direct comparison is inappropriate given that the force fields are not the same. From the plots of PMF (Fig. 2), we can see that the differential in $\Delta G_{b i n d}$ between 
SOPC and SDPC originates in the interfacial region $(z \approx 35 \AA)$ where $\alpha$ toc starts to interact with the membrane. An abrupt change in the slope of the PMF curve occurs there in SOPC, from flat when atoc is entirely in the aqueous phase to an approximately linear decline as the phytyl sidechain and then the chromanol group penetrates more deeply into the bilayer. In SDPC, the change in slope of the PMF curve is initially more gradual $(31 \leq z \leq 35 \AA)$ and only subsequently at greater depth within the bilayer does the curve become approximately linear in slope and falls approximately parallel to the curve in SOPC. We attribute this behavior to the more loosely packed nature of the polyunsaturated bilayer that reduces the thickness of the bilayer. The PMF curve in SDPC consequently lies a bit above that in SOPC, resulting in a correspondingly smaller binding energy.

\section{Flip-flop}

Between the equilibrium position $\left(z_{e q}\right)$ and the center of membrane $(z=0)$, there is an increase in the PMF which denotes a barrier to the transverse movement of atoc (Fig. 2). The barrier reflects that insertion of the hydrophilic $\mathrm{OH}$ group on the chromanol head group into the hydrophobic interior of the bilayer is energetically unfavorable. A plot (Figure S3, Supporting Information) of the number of water molecules within $5 \AA$ from the hydroxyl group in different simulation windows reveals desolvation accompanies the rise in free energy from $z_{\mathrm{eq}}(z \approx 10 \AA)$ to near the center of the bilayer $(z \approx 3 \AA)$, implying that the dehydration energy contributes to the flip-flop barrier. That the barrier in $\operatorname{SDPC}\left(\Delta G_{\text {flip }}=1.9 \mathrm{kcal} / \mathrm{mol}\right)$ is lower than in SOPC $\left(\Delta G_{\text {flip }}=2.5 \mathrm{kcal} / \mathrm{mol}\right)$ indicates that flip-flop of atoc from one side of the bilayer to the other is easier in the polyunsaturated than monounsaturated case (Table 1). A calculation of the rate of flip-flop now follows. 
To estimate the rate of flip-flop for atoc, we adopt an approach applied to cholesterol in the past. ${ }^{34}$ A complete flip-flop is defined as atoc moving from $z_{e q}$ (equilibrium position) in one leaflet to $z_{e q}$ in the other leaflet. In this process the molecule moves from $z_{e q}$ to the membrane center $(z=0)$ and then to $z_{e q}$ on the other side of the bilayer. The respective rate constants $k_{f}$ (from $z_{e q}$ on one side of the bilayer to $z=0$ ) and $k_{d}$ (from $z=0$ to $z_{e q}$ on the other side of the bilayer) that characterize these motions are related via

$$
k_{f}=k_{d} e^{-\frac{\Delta G_{f l i p}}{R T}},
$$

and equate to the rate of flip-flop (from $z_{e q}$ on one side to $z_{e q}$ on the other side of the bilayer)

$$
k_{\text {flip }}=\frac{1}{\left(k_{f}^{-1}+k_{d}^{-1}\right)} \times \frac{1}{2} .
$$

The factor of $1 / 2$ is included in eq. 3 to take into account the possibility that an atoc molecule that has reached the center of the membrane can return to its original leaflet as well as cross over to the other leaflet. To obtain an estimate of the value of $k_{d}, 10$ standard MD simulations were performed with atoc initially located at the center of membrane and the time $\left(t_{d}\right)$ it takes to move back to $z_{e q}$ without harmonic restraint was measured.

Table 2 lists the results of our calculations. They reveal that the average rate of flip-flop $\bar{k}_{\text {flip }}$ is approximately 3 times greater in SDPC $\left(\bar{k}_{\text {flip }}=7.5 \times 10^{5} \mathrm{~s}^{-1}\right)$ than SOPC $\left(\bar{k}_{\text {flip }}=\right.$ $\left.2.5 \times 10^{5} \mathrm{~s}^{-1}\right)$. The difference is qualitatively consistent with the greater number of flip-flop events observed for atoc in the two membranes in our previous standard simulations. ${ }^{22}$ Over 180 ns of simulation there were 22 flip-flops $\left(k_{\text {flip }} \sim 3 \times 10^{6} \mathrm{~s}^{-1}\right)$ in SDPC as opposed to just $2\left(k_{\text {flip }}\right.$ $\sim 3 \times 10^{5} \mathrm{~s}^{-1}$ ) in SOPC. The level of agreement with the values calculated for $\bar{k}_{f l i p}$ in the current study is reasonable given the somewhat rudimentary nature of the model used here and the poor statistics of an estimate based upon counting only a limited number of instances of flip-flop in 
the short duration of the earlier simulations.

\section{Membrane deformation}

Insight into the effect of desorption of $\alpha$ toc on membrane structure may be gleaned from inspection of the simulations obtained during umbrella sampling. Deformation of the SDPC and SOPC membrane is observed in the windows where atoc has been partly pulled out from the membrane. Figure 3A shows this deformation as a plot of $z(r)$, which is the average $z$ coordinate (relative to the center of then bilayer) of the central $\mathrm{C} 2$ atom on the glycerol backbone, against their lateral distance $r$ from atoc in different windows - i.e. atoc held at different depths in the membrane. The curves are mostly flat from window 0 , where the atoc molecule is at the middle of the bilayer, to widow 10, which is its equilibrium position. For windows 20 and 30, when $\alpha$ toc is in the interfacial region, nearby $(r \leq 15 \AA)$ there is an increase in $z(r)$. This indicates the phospholipid molecules located near atoc extend into water more than ones farther away - in other words, the pulling of atoc out of the bilayer deforms the membrane. The snapshot presented in Figure 3B gives an illustration of the deformation. Once atoc is completely in water, the membrane returns to normal as shown in window 35 where no deformation is apparent. We take the difference in $z(r)$ between the window $(r=5 \AA)$ where it is greatest in magnitude and where atoc is at its equilibrium depth as a measure of how much the membrane is deformed when atoc is removed. The difference is $4.7 \AA$ (between windows 30 and 10) in SDPC, which is less than the difference of $5.6 \AA$ in SOPC (between windows 31 and 11). Membrane deformation, we note, was also observed in other USMD simulations ${ }^{37}$ and has been applied to estimate the physical property of membranes. ${ }^{38}$ 


\section{Behavior at aqueous interface}

In the replica exchange method, it is possible that neighboring windows swap their replicas and cause them to take a random walk between the windows. ${ }^{39}$ In our simulations, we found examples of replicas that migrate through the interface between a window with $\alpha$ toc entirely in water and a window with atoc in contact with the membrane. The snapshots in Figure 4 give an illustration of the two states. With the trajectories of such replicas, we can take a close look at how atoc leaves the membrane into the water and, vice versa, enters the membrane from water. When atoc leaves the membrane, it begins from a state that has the chromanol head in the water and the phytyl tail is still inside the membrane and extends down into the interior (Fig. 4A). When atoc becomes fully pulled into the water (by replica exchange), the orientation of the chromanol and the whole molecule including the phytyl tail quickly becomes random and surrounded by water (Fig. 4B). On the other hand, when atoc enters the membrane from water, the phytyl chain encounters the membrane first. We did not find that water molecules penetrate into the membrane with the phytyl chain in this process.

Membrane deformation is also apparent when atoc enters the membrane from bulk water, which is illustrated in Figure S4 (Supporting Information) by snapshots of atoc re-inserting into the bilayer of SOPC from bulk water during the replica-exchange process (Fig. S4A). An accompanying plot of average $\mathrm{z}$ coordinate for the central $\mathrm{C} 2$ atom of the glycerol backbone in $\mathrm{SOPC}$ as a function of lateral distance from the atoc molecule, furthermore, indicates that the bilayer is flat before atoc contacts the membrane and then is deformed after the phytyl side chain re-inserts and interacts with the lipid (Fig. S4B). The amount of deformation is comparable to while atoc is at a similar depth when pulled out of the membrane. The implication is that the deformation is energetically favorable for the whole system when atoc is partially inserted into 
the membrane, presumably due to the atoc-PC interactions.

\section{Chromanol orientation}

How the orientation of the chromanol group on atoc changes with depth in the membrane was mapped with a two-dimensional probability histogram $\rho\left(z, r_{z}\right)$. The orientation is represented by the tilt of the chromanol ring $r_{z}$, as defined by $r_{z} \equiv \cos (\theta)$ where $\theta$ is the angle that the C3-C6 vector on the chromanol group makes with the bilayer normal (Fig. 5A). It can be shown that if the orientation is completely random and uniform in 3-dimensional space, $r_{z}$ will obey a uniform distribution between -1 and $+1 . z$ and $r_{z}$ are divided into bins that are $0.5 \AA$ and 0.02 in respective size. The values of $\rho\left(z, r_{z}\right)$ at each $z$ are normalized so that they sum to one.

The two-dimensional histograms of orientation for the chromanol group obtained in SDPC and SOPC are plotted in Figure 5B. They are similar in the two membranes. As would be expected, the chromanol group has no preferred direction when the entire atoc molecule is in water $(z>35 \AA)$. When the phytyl chain penetrates the bilayer $(z<35 \AA)$, it packs parallel to the surrounding phospholipid chains and the orientation of the chromanol group becomes constrained in a distribution that tends to line up with the bilayer normal $\left(r_{z}=1, \theta=0^{\circ}\right)$. Near the disordered center of the bilayer $(z \sim 0 \AA)$, however, the situation changes and the orientation becomes almost uniformly distributed again. It indicates that atoc would undergo a lot of reorientation as it crosses the membrane, and that our simulations have a good sampling of the orientation of the chromanol.

\section{DISCUSSION}

Vitamin E binds less strongly to a polyunsaturated phospholipid 
The role of atoc in the plasma membrane is to protect polyunsaturated phospholipids from oxidation, an activity that requires close contact with the lipid peroxyl radicals formed during the chain reaction by which oxidation proceeds. Because the global concentration of atoc is small ( $<1 \mathrm{~mol} \%$ of all lipids) ${ }^{16}$ how it can effectively protect the comparatively larger amount of polyunsaturated phospholipid remains a question. ${ }^{15}$ The answer may lie in the unique membrane properties of PUFA chains that contain multiple $-\mathrm{C}-\mathrm{C}=\mathrm{C}-\mathrm{C}-$ units. The $\mathrm{C}-\mathrm{C}$ bonds next to each $\mathrm{C}=\mathrm{C}$ bond have a shallow rotational energy barrier, so that PUFA chains are tremendously disordered. ${ }^{40}$ This disorder provides a membrane environment necessary for the function of specific proteins. ${ }^{41}$ It also produces an aversion for cholesterol that drives the formation of PUFA-rich domains depleted in the sterol, ${ }^{42}$ leading to the hypothesis that atoc preferentially co-localizes with PUFA in such domains to increase the local concentration of atoc around the lipid species most in need of protection from oxidation. ${ }^{15}$

Our simulations appear to contradict the proposal that atoc and polyunsaturated phospholipids preferentially interact with each other. They reveal that there is a higher binding energy for atoc associated with monounsaturated SOPC $(18.3 \mathrm{kcal} / \mathrm{mol})$ than with polyunsaturated SDPC $(16.7 \mathrm{kcal} / \mathrm{mol})($ Table 1$)$. A partition coefficient $K_{S D P C}^{S O P C}=10.9(\mathrm{SOPC}$ with respect to SDPC), correspondingly, was evaluated from the profile of PMF (Fig. 2) by integrating the probabilities associated with $G(z)$ via

$$
K_{S D P C}^{S O P C}=\frac{\int e^{-\frac{G_{S O P C}(z)}{R T}} d z}{\int e^{-\frac{G_{S D P C}(z)}{R T}} d z} .
$$

The limits of the integrals are between $z=0$ (atoc at the center of the membrane) and the value of $z$ where the profile levels off (atoc no longer in membrane), while $R$ and $T$ are respectively the gas constant and temperature. These results imply that were SDPC and SOPC domains (hypothetically) to exist in a membrane, atoc would prefer SOPC over SDPC - i.e. the 
polyunsaturated domain would not be favored. A somewhat analogous preference for saturated over unsaturated PC was found in coarse grain (CG) MD simulations run on vitamin E added to a binary mixture of 1,2-dipalmitoylphosphatidylcholine (DPPC, 16:0-16:0PC) and 1,2dilinoleoylphosphatidylcholine (18:2-18:2PC). ${ }^{43}$ However, the situation is far more complex in a biological membrane. Plasma membranes contain cholesterol, sometimes $>50 \mathrm{~mol} \%$ of total lipid, ${ }^{44}$ with an affinity for predominantly saturated sphingolipids that drives segregation into sphingolipid-rich/cholesterol-rich domains (lipid rafts). ${ }^{45}$ We postulate that the high concentration of cholesterol in these domains will exclude atoc into PUFA-rich/sterol-poor (nonraft) regions. To test this possibility, we plan to perform UBSMD simulations comparing the binding energy of atoc for bilayers in the presence of cholesterol. CG simulations that can reproduce the heterogeneous distribution of lipids in a membrane ${ }^{46}$ offer another approach that we plan to try to directly observe the partitioning of atoc between domains.

The trend we saw here for the binding of atoc is the same as seen for cholesterol. Greater affinity for saturated than unsaturated lipids has been measured for cholesterol by experiment ${ }^{47,48}$ and USMD simulation. ${ }^{33,34}$ Upon reflection, this observation is not too surprising given the similarity in molecular structure of cholesterol and $\alpha$ toc. ${ }^{15}$ Both molecules possess a rigid moiety with a hydroxyl group, which usually anchors at the aqueous interface, at one end and a hydrophobic chain, which extends to extend towards the middle of the bilayer, at the opposite end. Like in the case of cholesterol, the more disordered lipid environment in a polyunsaturated bilayer is responsible for the differences in binding energy for atoc. SDPC is thinner and more loosely packed (larger area per lipid) than SOPC. ${ }^{22}$ Thus, in the plot of PMF the change of $G(z)$ is more gradual at the aqueous interface ( $z$ between $30-35 \AA$ ), as well as near the center of membrane (from $z_{e q}$ to $z=0$ ), for atoc in SDPC compared to SOPC (Fig. 2). 
Our USMD simulations furnish further details of the desorption process. In the windows where atoc is sticking out from the membrane, we noticed deformation of the membrane (Fig. 3). The implication is that not only atoc-phosphatidylcholine (PC) interactions, but also PC-PC interactions, contribute to the binding energy $\Delta G_{b i n d}$. Snapshots from the replica exchange method also reveal the strongly hydrophobic nature of the behavior of atoc near the aqueous interface (Fig. 4). The water layer that surrounds an atoc molecule when it is in water does not follow it into the bilayer hydrophobic interior. This avoids the possibility that a water pore forms during the simulation, which might affect the accuracy of the PMF by perturbing membrane order. $^{49}$

\section{Vitamin E flip-flops more often in a polyunsaturated bilayer}

Flip-flop of phospholipids across protein-free membranes is slow because the hydrophobic interior of a lipid bilayer is an energetically unfavorable environment for hydrophilic head groups. ${ }^{50}$ The timescale of the process is hours or longer, depending upon head group and chain composition. ${ }^{51}$ Reflecting the faster flip-flop that accompanies increased unsaturation, the rate measured is $40 \%$ greater for SDPC vs. SOPC..$^{52}$ Few estimates of the rate of flip-flop exist for atoc. ${ }^{16}$ A more rapid timescale of seconds or less, akin to cholesterol, ${ }^{53}$ is the growing consensus in recent work. ${ }^{20-22}$

The smaller energy barrier at the center of the bilayer identified in the profile of PMF for SDPC than SOPC (Fig. 2) shows that it is easier for atoc to translocate across a thinner, highly disordered polyunsaturated membrane. This assessment is confirmed by the faster rate of flipflop calculated for SDPC than SOPC (Table 2), and agrees with the higher incidence of flip-flop observed for atoc in SDPC vs. SOPC in our previous standard simulations. ${ }^{22}$ The same trend of 
faster flip-flop in more unsaturated, disordered membranes was seen for cholesterol in MD simulations. ${ }^{34,54,55}$ Fast flip-flop for atoc in SDPC implies the vitamin could easily penetrate the interior of a polyunsaturated bilayer to intercept lipid peroxyl radicals, which would help in the protection against oxidation. ${ }^{22}$ Our simulations also provide insight on the path that the flip-flop of atoc takes. An upright orientation that remains lined up with the bilayer normal applies to the chromanol group throughout most of the bilayer in both SDPC and SOPC (Fig. 5B). In the vicinity middle of the bilayer, in contrast, the orientation becomes random so that the chromanol group can invert and place the $\mathrm{OH}$ group towards the aqueous interface on the opposite side of the membrane. This scenario is similar to that envisaged for the flip-flop of cholesterol in lipid membranes studied by the USMD method. ${ }^{34,54}$

\section{Concluding remarks}

In summary, our USMD simulations demonstrate that the dynamics and organization of vitamin E in many respects resemble that of structurally similar cholesterol. Paralleling the behavior of cholesterol in phospholipid bilayers, the binding energy of atoc is lower and its flipflop is faster with increased acyl chain unsaturation. Our findings do not support the proposal that vitamin E binds preferentially to polyunsaturated phospholipids. We postulate that the overwhelming higher concentration of cholesterol found in an animal plasma membrane, however, may cause atoc to co-localize with polyunsaturated phospholipids. According to this idea, cholesterol displaces atoc from regions enriched in saturated lipids for which the sterol has high affinity into regions enriched in polyunsaturated lipids for which the sterol has poor affinity.

\section{ASSOCIATED CONTENT}




\section{Supporting Information}

Additional results derived from simulations.

\section{ACKNOWLEDGEMENTS}

This work was supported by the School of Science, IUPUI. The simulations were performed on the Big Red II supercomputer at Indiana University. 


\section{REFERENCES}

(1) Zingg, J. M. Vitamin E: an overview of major research directions. Mol. Aspects Med. 2007, 28, 400-422.

(2) Niki, E.; Traber, M. G. A history of vitamin E. Ann. Nutr. Metab. 2012, 61, 207-212.

(3) Ulatowski, 1; Manor, D. Vitamin E trafficking in neurologic health and disease. Ann. Rev. Nutr. 2013, 33, 87-103.

(4) Packer, L. Vitamin E is nature's master antioxidant. Sci. Am. Sci. Med. 1994, 1, 54-63.

(5) Wang, X.; Quinn, P. J. Vitamin E and its function in membranes. Prog. Lipid Res. 1999, 38, 309-336.

(6) Traber, M. G.; Atkinson, J. Vitamin E, antioxidant and nothing more. Free Rad. Biol. Med. 2007, 43, 4-15.

(7) Calder, P. C. Marine omega-3 fatty acids and inflammatory processes: Effects, mechanisms and clinical relevance. Biochim. Biophys. Acta 2015, 1851, 469-484.

(8) Hou, T. Y.; McMurray, D. N.; Chapkin, R. S. Omega-3 fatty acids, lipid rafts, and T cell signaling. Eur. J. Pharmacol. 2016, 785, 2-9.

(9) Shaikh, S. R. Biophysical and biochemical mechanisms by which dietary N-3 polyunsaturated fatty acids from fish oil disrupt membrane lipid rafts. J. Nutr. Biochem. 2012, $23,101-105$.

(10) Raederstorff, D.; Wyss, A.; Calder, P. C.; Weber, P.; Eggersdorfer, M. Vitamin E function and requirements in relation to PUFA. Brit. J. Nutr. 2015, 114, 1113-1122.

(11) Burton, G. W.; Ingold, K. U. Vitamin E: application of the principles of physical organic chemistry to the exploration of its structure and function. Acc. Chem. Res. 1986, 19, 194-201.

(12) Alessi, M.; Paul, T.; Scaiano, J. C.; Ingold, K. U. The contrasting kinetics of peroxidation 
of vitamin E-containing phospholipid unilamellar vesicles and human low-density lipoprotein. $J$. Am. Chem. Soc. 2012, 124, 6957-6965.

(13) Diplock, A. T.; Lucy, J. A. The biochemical modes of action of vitamin E: a hypothesis. FEBS Lett. 1973, 29, 205-210.

(14) Kagan, V. E. Tocopherol stabilizes membranes against phospholipase A, free fatty acids, and lysophospholipids. Annals. NY Acad. Sci. 1989, 570, 121-135.

(15) Atkinson, J.; Harroun, T.; Wasssall, S. R.; Stillwell, W.; Katsaras, J. The location and behavior of $\alpha$-tocopherol in membranes. Mol. Nutr. Food Res. 2010, 54, 641-651.

(16) Atkinson, J.; Epand, R. F.; Epand, R. M. Tocopherols and tocotrienols in membranes: a critical review. Free Rad. Biol. Med. 2008, 44, 739-764.

(17) Marquardt, D.; Kučerka, N.; Katsaras, J.; Harroun, T. A. $\alpha$-Tocopherol's location in membranes is not affected by their composition. Langmuir 2015, 31, 4464-4472.

(18) Ausili, A.; de Godos, A. M.; Torrecillas, A.; Aranda, F. J.; Corbalán-García, S.; GómezFernández, J. C. The vertical location of $\alpha$-tocopherol in phosphatidylcholine membranes is not altered as a function of the degree of unsaturation of the fatty acyl chains. Phys. Chem. Chem. Phys. 2017, 19, 6731-6742.

(19) Marquardt, D.; Williams, J. A.; Kučerka, N.; Atkinson, J.; Wassall, S. R.; Katsaras, J.; Harroun, T. A. Tocopherol activity correlates with its location in a membrane: a new perspective on the anti-oxidant vitamin E. J. Am. Chem. Soc. 2013, 135, 7523-7533.

(20) Qin, S-S.; Yu, Z-W.; Yu, Y-X. Structural and kinetic properties of $\alpha$-tocopherol in phospholipid bilayers, a molecular dynamics simulation study. J. Phys. Chem. B 2009, 113, 16537-16546.

(21) Qin, S-S.; Yu, Z-W. Molecular dynamics simulations of $\alpha$-tocopherol in model 
membranes. Acta Phys. - Chim. Sin. 2011, 27, 213-227.

(22) Leng, X.; Kinnun, J. J.; Marquardt, D.; Ghefli, M.; Kucerka, N.; Katsaras, J.; Atkinson, J.; Harroun, T. A.; Feller, S. E.; Wassall, S. R. $\alpha$-Tocopherol is well designed to protect polyunsaturated phospholipids: MD simulations. Biophys. J. 2015, 109, 1608-1618.

(23) Leng, X.; Kinnun, J. J.; Cavazos, A. T.; Canner, S. W.; Shaikh, S. R.; Feller, S. E.; Wassall, S. R. All n-3 PUFA are not the same: MD simulations reveal differences in membrane organization for EPA, DHA and DPA. Biochim. Biophys. Acta 2018, 1860, 1125-1134.

(24) Klauda, J.B.; Monje, V.; Kim, T.; Im, W. Improving the CHARMM force field for polyunsaturated fatty acid chains. J. Phys Chem. B 2012, 116, 9424-9431.

(25) Darden, T.; York, D.; Pedersen, L. Particle mesh Ewald: an $n \cdot \log (n)$ method for Ewald sums in large systems. J. Chem. Phys. 1993, 98, 10089-10092.

(26) Feller, S. E.; Zhang, Y.; Pastor, R. W.; Brooks, R. B. Constant pressure molecular dynamics simulation: the Langevin piston method. J. Chem. Phys. 1995, 103, 4613-4621.

(27) Bereau, T.; Swendsen, R. H. Optimized convergence for multiple histogram analysis. $J$. Comp. Phys. 2009, 228, 6199-6129.

(28) Fukunishi, H.; Watanabe, O.; Takada, S. On the Hamiltonian replica exchange method for efficient sampling of biomolecular systems: application to protein structure prediction. J. Chem. Phys. 2002, 116, 9058-9067.

(29) Phillips, J. C.; Braun, R.; Wang, W.; Gumbart, J.; Tajkhorshid, E.; Villa, E.; Chipot, C.; Skeel, R. D.; Kalé, L.; Schulten, K. Scalable molecular dynamics with NAMD. J. Comp. Chem. 2005, 26, 1781-1802.

(30) Kumar, S.; Bouzida, D.; Swendsen, R. H.; Kollman, P. A.; Rosenberg, J. M. The weighted histogram analysis method for free-Energy calculations on biomolecules 1. The 
method. J. Comp. Chem. 1992, 13, 1011-1021.

(31) Zhu, F.; Hummer, G. Convergence and error estimation in free energy calculations using the weighted histogram analysis method. J. Comp. Chem. 2012, 33, 453-465.

(32) Flyvbjerg, H.; Petersen, H. G. Error-estimates on averages of correlated data. J. Chem. Phys. 1989, 91, 461-466.

(33) Zhang, Z.; Lu, L.; Berkowitz, M. L. Energetics of cholesterol transfer between lipid bilayers. J. Phys. Chem. B 2008, 112, 3807-3811.

(34) Bennett, W. D.; MacCallum, J. L.; Hinner, M. J.; Marrink, S. J.; Tieleman, D. P. Molecular view of cholesterol flip-flop and chemical potential in different membrane environments. J. Am. Chem. Soc. 2009, 131, 12714-12720.

(35) Meng, F. Molecular simulation of $\alpha$-tocopherol passing across DPPC lipid using potential of mean force and accelerated molecular dynamics method. J. Theor. Comput. Chem. 2013, 12, 1341011-1-1341011-16.

(36) Boonnoy, P.; Karttunen, M.; Wong-ekkabut, J. Alpha-tocopherol inhibits pore formation in oxidized bilayers. Phys. Chem. Chem. Phys. 2017, 19, 5699-5704.

(37) Neale, C.; Pomes, R Sampling errors in free energy simulations of small molecules in lipid bilayers. Biochim. Biophys. Acta 2016, 1858, 2539-2548.

(38) Pieffet, G.; Botero, A.; Peters, G. H.; Forero-Shelton, M.; Leidy, C. Exploring the local elastic properties of bilayer membranes using molecular dynamics simulations. J. Phys. Chem. B 2014, $118,12883-12891$.

(39) Sugita, Y.; Kitao, A.; Okamoto, Y. Multidimensional replica-exchange method for freeenergy calculations. J. Chem. Phys. 2000, 113, 6042-6051.

(40) Feller, S. E. Acyl chain conformations in phospholipid bilayers: a comparative study of 
docosahexaenoic acid and saturated fatty acids. Chem. Phys. Lipids 2008, 153, 76-80.

(41) Grossfield, A.; Feller, S. E.; Pitman, M. C. Contribution of omega-3 fatty acids to the thermodynamics of membrane protein solvation. J. Phys. Chem. B 2006, 110, 8907-8909.

(42) Wassall, S. R.; Stillwell, W. Polyunsaturated fatty acid-cholesterol interactions: domain formation in membranes. Biochim. Biophys. Acta 2009, 1788, 24-32.

(43) Muddana, H. S.; Chiang, H. H.; Butler, P. J. Tuning membrane phase separation using nonlipid amphiphiles. Biophys. J. 2012, 102, 489-497.

(44) Stillwell, W. An Introduction to Biological Membranes (Second Edition). Elsevier Science, Oxford, UK, 2016.

(45) Lingwood, D.; Simons, K. Lipid rafts as a membrane-organizing principle. Science 2010, $327,46-50$.

(46) Risselada, H. J.; Marrink, S. J. The molecular face of lipid rafts in model membranes. Proc. Natl. Acad. Sci. 2008, 105, 17367-17372.

(47) Nui, S. L.; Litman, B. J. Determination of membrane cholesterol partition coefficient using a lipid vesicle-cyclodextrin binary system: effect of phospholipid acyl chain unsaturation and headgroup composition. Biophys. J. 2002, 83, 3408-3415.

(48) Williams, J. A.; Wassall, C. D.; Kemple, M. D.; Wassall, S. R. An EPR method for measuring the affinity of a spin labeled analog of cholesterol for phospholipids. J. Membrane Biol. 2013, 246, 689-696.

(49) Neale, C.; Madill, C.; Rauscher, S.; Pomès, R. Accelerating convergence in molecular dynamics simulations of solutes in lipid membranes by conducting a random walk along the bilayer normal. J. Chem. Theor. Comput. 2013, 9, 3686-3703.

(50) Contreras, F.-X.; Sánchez-Magraner, L.; Alonso, A.; Goni, F. M. Transbilayer (flip-flop) 
lipid motion and lipid scrambling in membranes. FEBS Lett. 2010, 584, 1779-1786.

(51) Homan, R; Pownall, H. J. Transbilayer diffusion of phospholipids: dependence on headgroup structure and acyl chain length. Biochim. Biophys. Acta 1998, 938, 155-166.

(52) Armstrong, V. T.; Brzustowicz, M. R.; Wassall, S. R.; Jenski, L. J.; Stillwell, W. Rapid flip-flop in polyunsaturated (docosahexaenoate) phospholipid membranes. Arch. Biochem. Biophys. 2003, 414, 74-82.

(53) Hamilton, J. A. Fast flip-flop of cholesterol and fatty acids in membranes: Implications for membrane transport proteins. Curr. Opin. Lipidol. 2003, 14, 263-271.

(54) Jo, S.; Rui, H.; Lim, J. B.; Klauda, J. B.; Im, W. Cholesterol flip-flop: insights from free energy simulation studies. J. Phys. Chem. B 2010, 114, 13342-13348.

(55) Bennett, W. F. D; Tieleman, D. P Molecular simulation of rapid translocation of cholesterol, diacylglycerol, and ceramide in model raft and nonraft membranes. J. Lipid Res. 2012, 53, 421-429. 
Table 1 Energetic terms derived from the PMF profile of atoc.

\begin{tabular}{c|ccc} 
& $\begin{array}{c}\text { Binding Energy } \\
(\mathrm{kcal} / \mathrm{mol})\end{array}$ & $\begin{array}{c}\text { Flip-flop barrier } \\
(\mathrm{kcal} / \mathrm{mol})\end{array}$ & $\begin{array}{c}\text { Equilibrium depth } \\
(\AA)\end{array}$ \\
\hline \hline SDPC & $16.7 \pm 0.3$ & $1.9 \pm 0.2$ & 10.1 \\
SOPC & $18.3 \pm 0.4$ & $2.5 \pm 0.1$ & 11.4
\end{tabular}


Table 2 Kinetic properties of atoc flip-flop in SDPC and SOPC at $310 \mathrm{~K}$. Data are taken from the average of 20 cases ( 2 atoc in 10 standard MD simulations) - 16/20 cases in SDPC and $15 / 20$ cases in SOPC (atoc did not reach equilibrium within $60 \mathrm{~ns}$ in the rest of the cases). The range of $t_{d}$ and derived rate constants are shown for reference, as well as the average rate of flipflop.

\begin{tabular}{c|c|c|c|c|c} 
& $t_{d}(\mathrm{~ns})$ & $k_{d}\left(\mathrm{~s}^{-1}\right)$ & $k_{f}\left(\mathrm{~s}^{-1}\right)$ & $k_{\text {flip }}\left(\mathrm{s}^{-1}\right)$ & $\bar{k}_{\text {flip }}\left(\mathrm{s}^{-1}\right)$ \\
\hline \hline SDPC & 8 to 55 & $1.3 \times 10^{8}$ to & $5.7 \times 10^{6}$ to & $2.7 \times 10^{6}$ to & $7.5 \times 10^{5}$ \\
& & $1.8 \times 10^{7}$ & $8.3 \times 10^{5}$ & $4.0 \times 10^{5}$ & \\
\hline SOPC & 8 to 60 & $1.3 \times 10^{8}$ to & $2.2 \times 10^{6}$ to & $1.1 \times 10^{6}$ to & $2.5 \times 10^{5}$ \\
& & $1.7 \times 10^{7}$ & $2.9 \times 10^{5}$ & $1.4 \times 10^{5}$ &
\end{tabular}




\section{FIGURE LEGENDS}

Figure $1 \quad$ Molecular structure of $\alpha$ toc, SDPC and SOPC.

Figure 2 PMF for atoc partitioning from water to the center of the bilayer in SDPC (red) and SOPC (green). The PMF was set to zero in the aqueous phase (Fig. S1 in Supporting Information depicts an alternative alignment with the PMF set to zero at $z_{e q}$ ). Error bars are the standard error calculated using block averaging. Supporting Information, in addition, includes two-dimensional PMF (in $\mathrm{z}$ and $r_{z} \equiv \cos (\theta)$ to represent orientation) that were also calculated from our 1-dimensional sampling data (Fig. S2).

Figure 3 A. Average $\mathrm{z}$ coordinate (with maximum discrepancy of $0.4 \AA$ ) of the central $\mathrm{C} 2$ atom of the glycerol backbone in PC lipids as a function of lateral distance $\mathrm{r}$ from the $\alpha$ toc molecule at in different windows (different depths of the chromanol group).

B. Snapshot of the system when $\alpha$ toc has been half-pulled out from the membrane (SDPC). aToc is shown as a space-filling model, the head groups of PC lipid are shown in an orange surface representation and the acyl chains of PC lipids are shown as blue lines.

Figure 4 Snapshots from the trajectory of one replica that swaps between the windows of atoc in and out of the membrane - atoc with the chromanol group restrained in the water and the phytyl chain inside the membrane (A), and with the whole molecule completely in water (B). aToc is shown as a space-filling model (chromanol group colored in light blue and phytyl chain colored in orange), water molecules within a distance of $5 \AA$ from atoc are shown as blue balls, and PC lipids with their head group within a lateral distance of $10 \AA$ from atoc are shown in 
licorice representation.

Figure 5 Probability distribution for the orientation of the chromanol group on atoc (A) as a function of depth in SDPC and SOPC (B). The orientation is denoted by $r_{z} \equiv \cos (\theta)$, where $\theta$ is the angle that the $\mathrm{C} 3-\mathrm{C} 6$ vector on the chromanol group makes with the bilayer normal. 
atoc
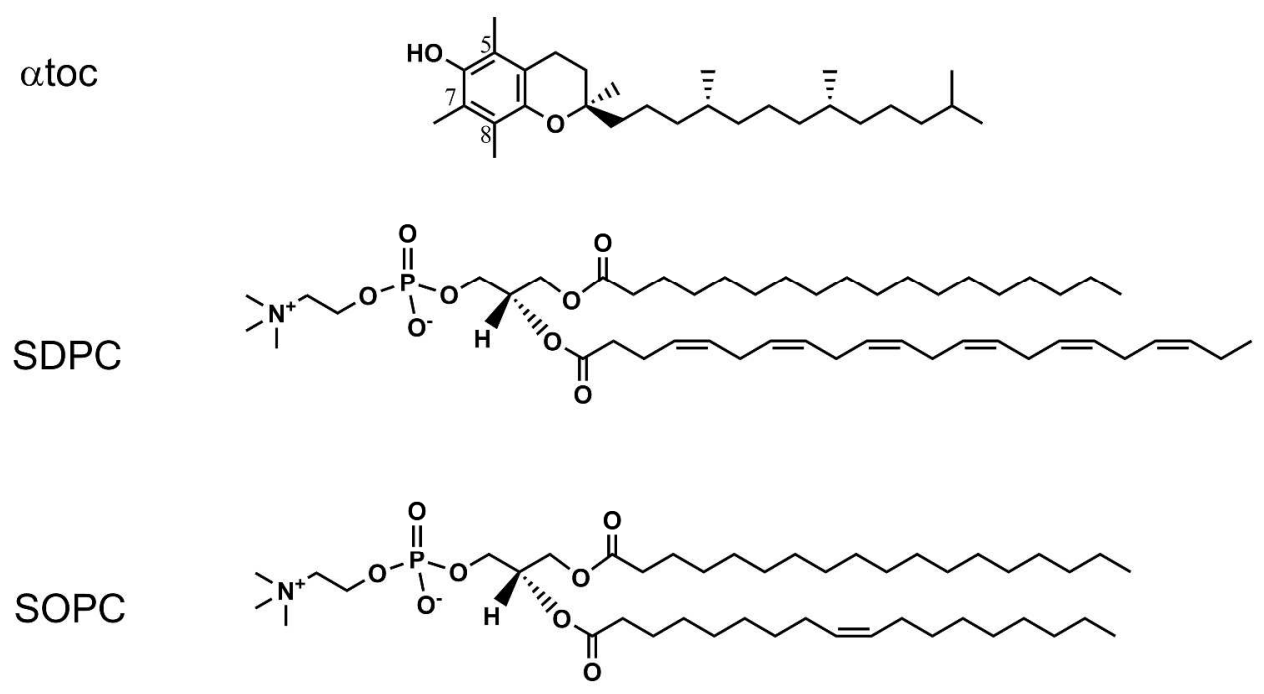

Figure 1 


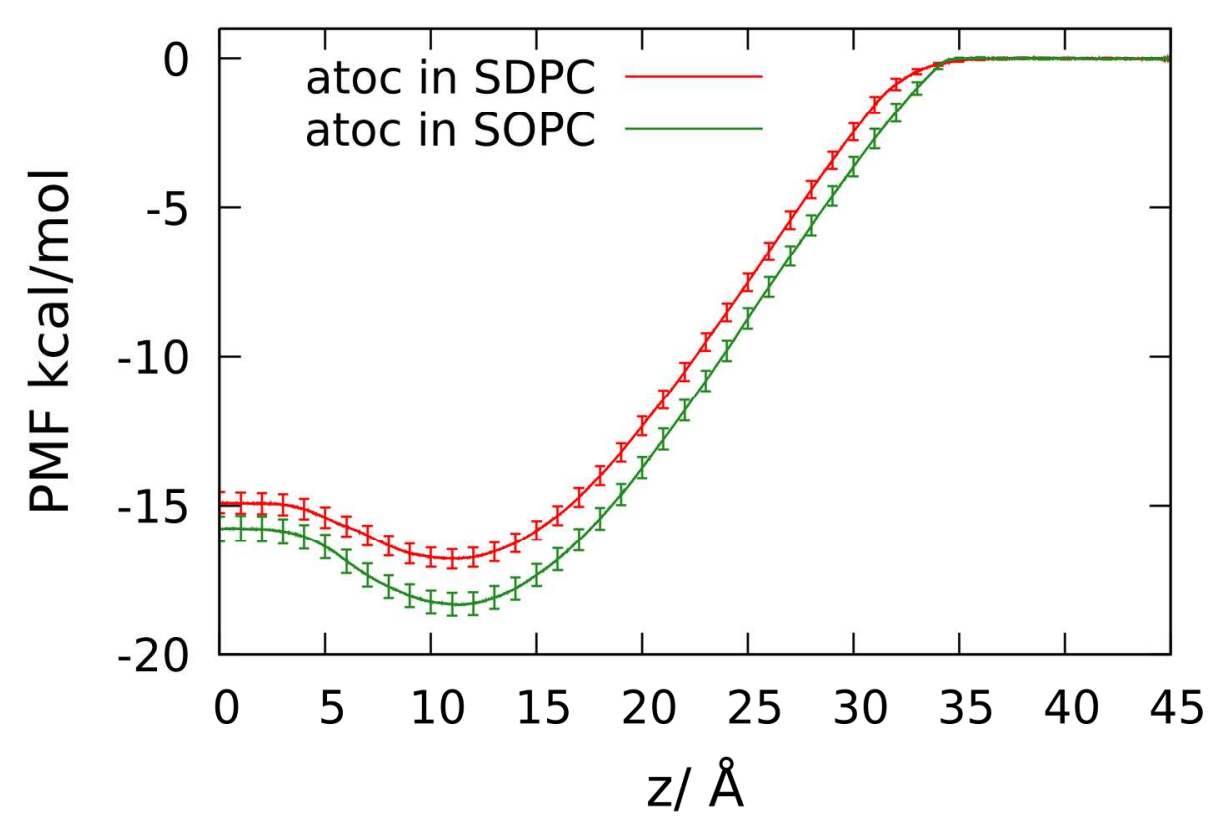

Figure 2

59

60

ACS Paragon PTus Environment 
A

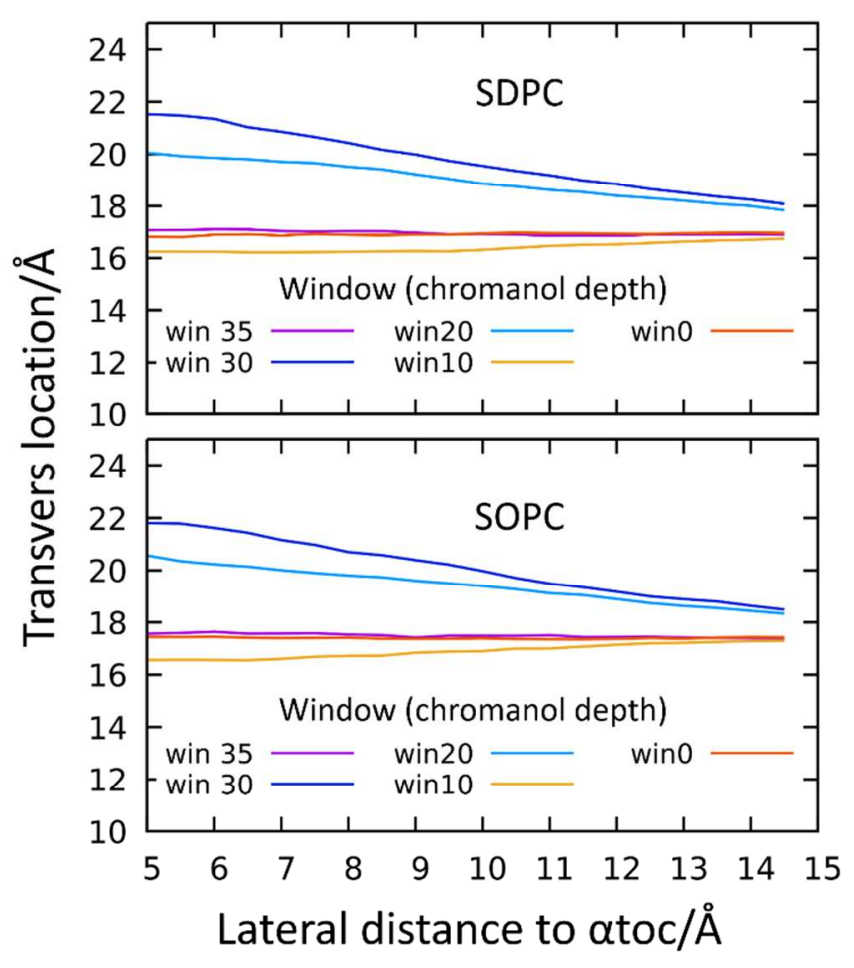

B

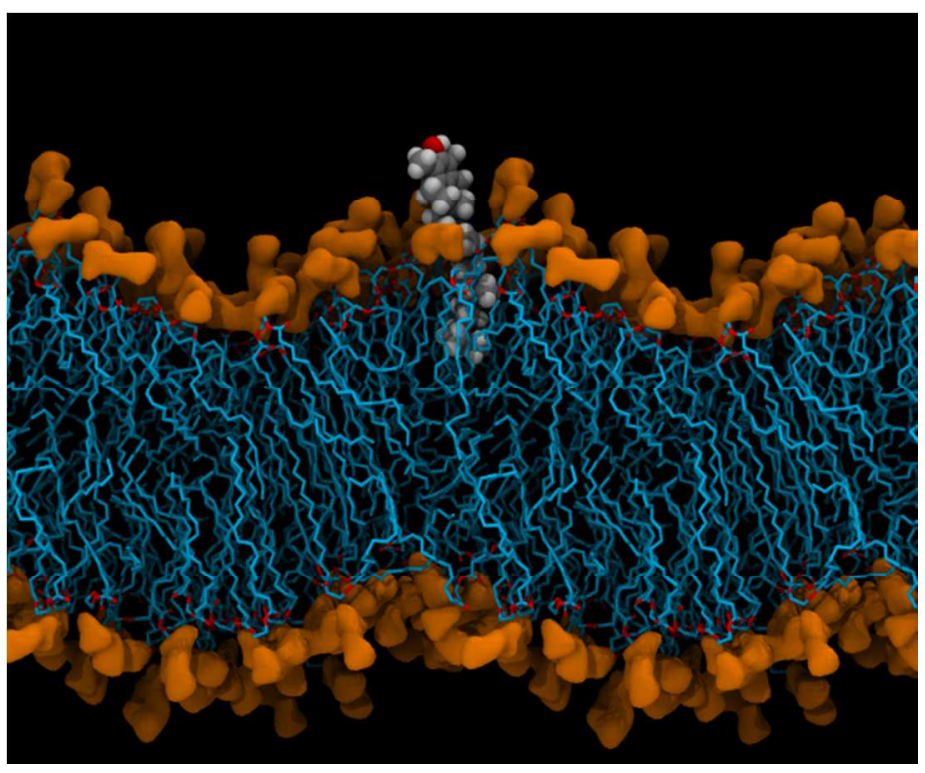

Figure 3 
A B

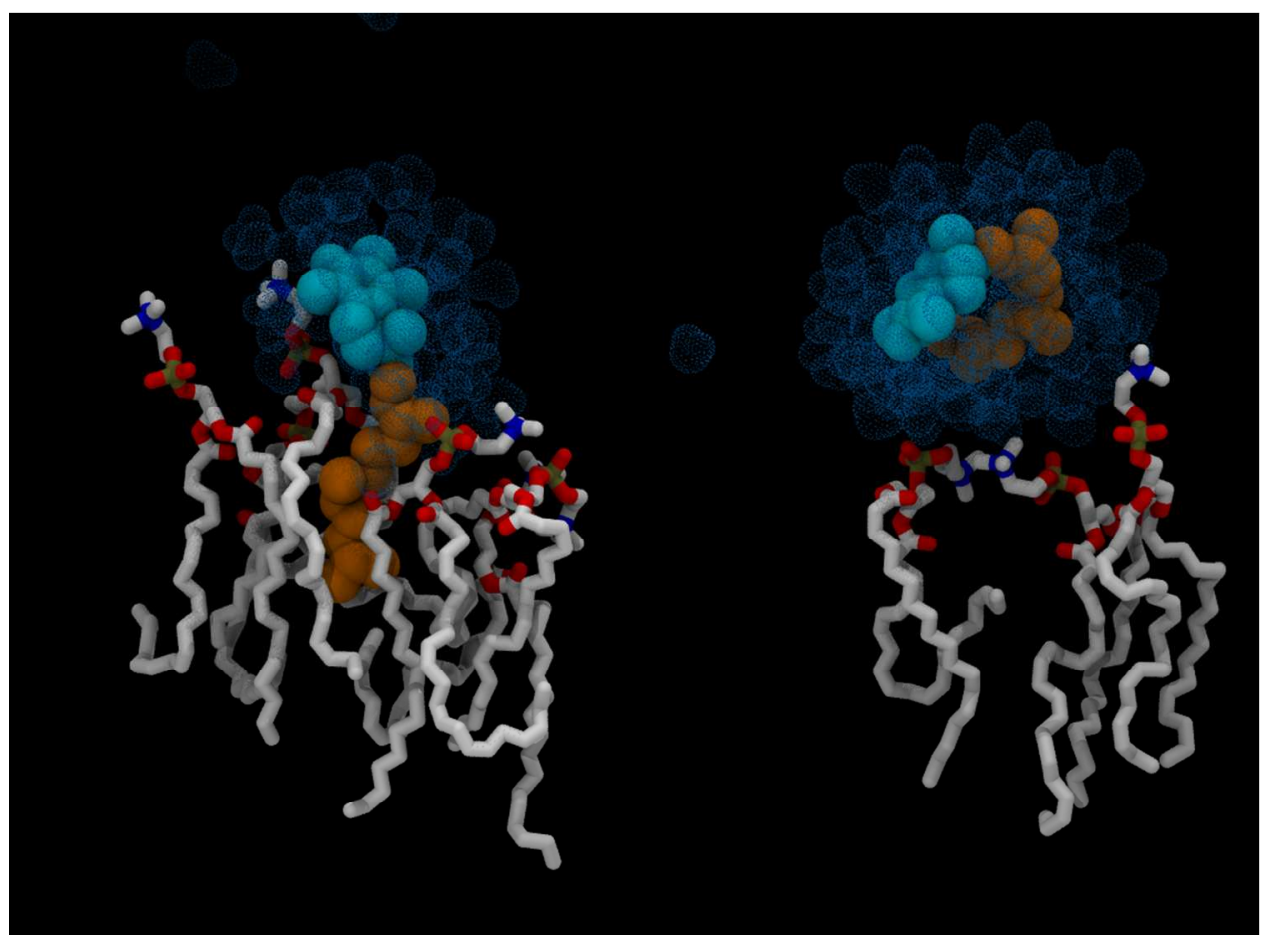

Figure 4 
A

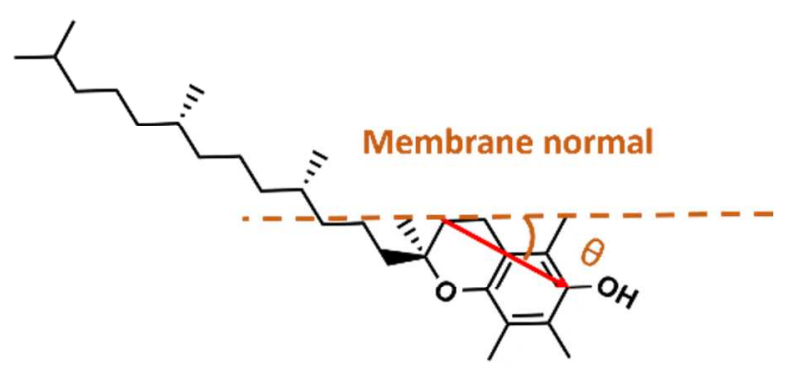

B

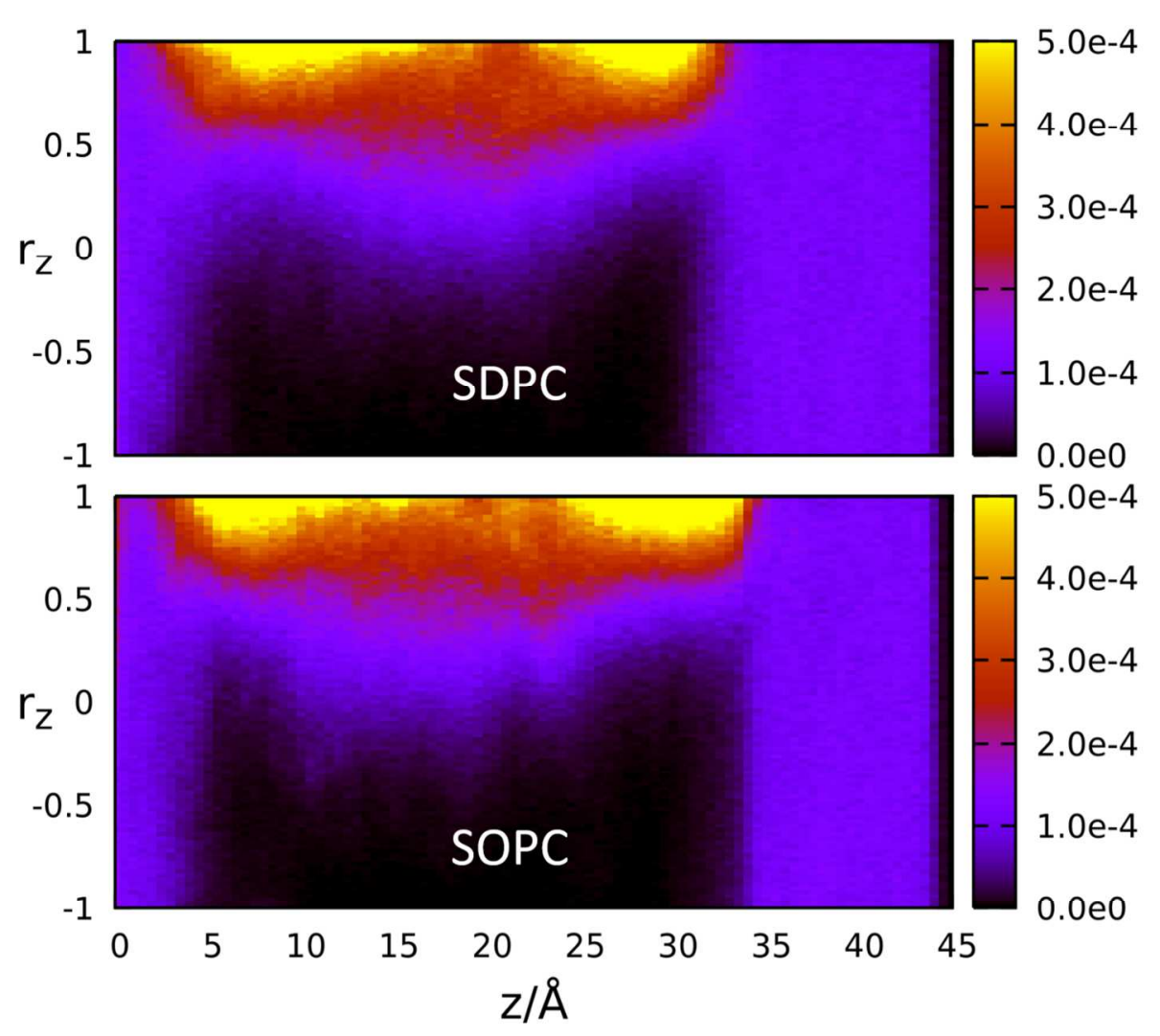

Figure 5 


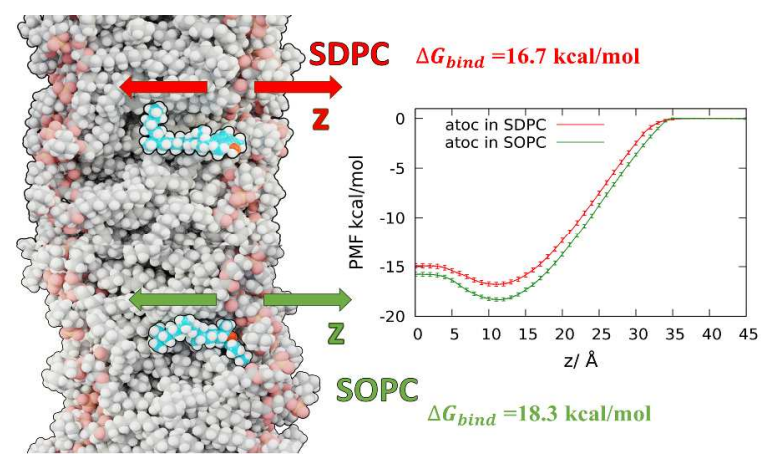

Toc Graphic 
atoc
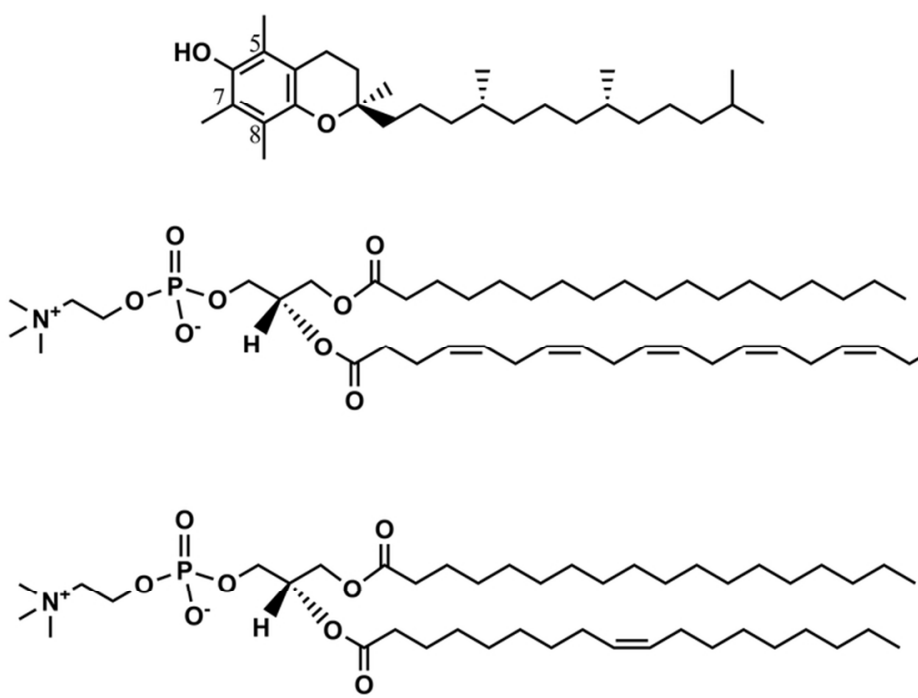

Figure 1. Molecular structure of atoc, SDPC and SOPC.

$46 \times 26 \mathrm{~mm}(600 \times 600 \mathrm{DPI})$ 


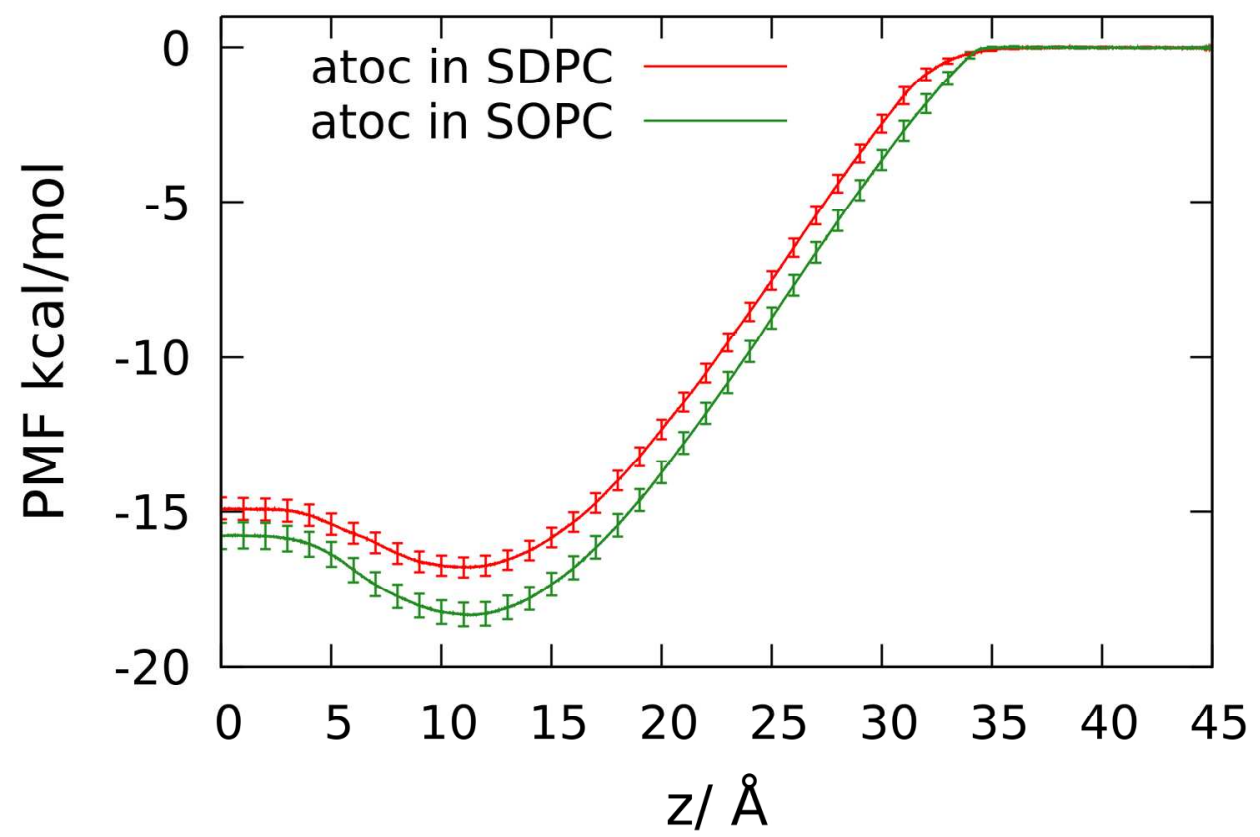

PMF for atoc partitioning from water to the center of the bilayer in SDPC (red) and SOPC (green). The PMF was set to zero in the aqueous phase (Fig. S1 in Supporting Information depicts an alternative alignment with the PMF set to zero at z_eq). Error bars are the standard error calculated using block averaging. Supporting Information, in addition, includes two-dimensional PMF (in $z$ and $r_{-} z \equiv \cos (\theta)$ to represent orientation) that were also calculated from our 1-dimensional sampling data (Fig. S2).

$776 \times 529 \mathrm{~mm}(72 \times 72 \mathrm{DPI})$ 
Figure 3. (A). Average $z$ coordinate (with maximum discrepancy of $0.4 \AA$ ) of the central C2 atom of the glycerol backbone in PC lipids as a function of lateral distance $r$ from the atoc at in different windows (different depths of the chromanol group). (B). Snapshot of the system when atoc has been half-pulled out from the membrane (SDPC). aToc is shown as a space-filling model, the head groups of PC lipid are shown in an orange surface representation and the acyl chains of PC lipids are shown as blue lines.

$$
146 \times 260 \mathrm{~mm} \text { ( } 300 \times 300 \text { DPI) }
$$


Figure 4. Snapshots from the trajectory of one replica that swaps between the windows of atoc in and out of the membrane - atoc with the chromanol group restrained in the water and the phytyl chain inside the membrane $(A)$, and with the whole molecule completely in water $(B)$. aToc is shown as a space-filling model (chromanol group colored in light blue and phytyl chain colored in orange), water molecules within a distance of $5 \AA$ from atoc are shown as blue balls, and PC lipids with their head group within a lateral distance of $10 \AA$ from atoc are shown in licorice representation.

$60 \times 44 \mathrm{~mm}(300 \times 300 \mathrm{DPI})$ 
A
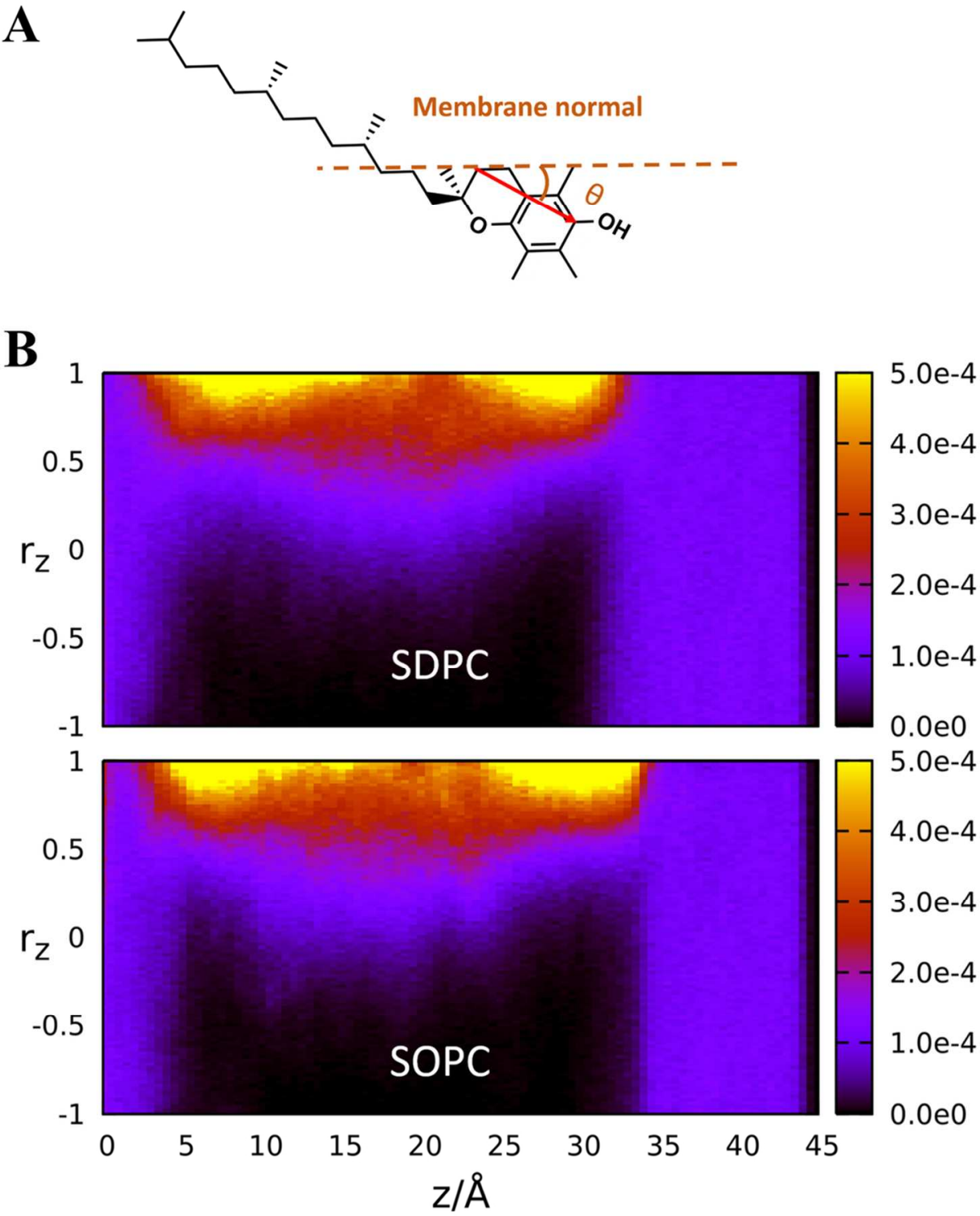

Figure 5. Probability distribution for the orientation of the chromanol group on atoc $(A)$ as a function of depth in SDPC and SOPC (B). The orientation is denoted by $r_{-} z \equiv \cos (\theta)$, where $\theta$ is the angle that the C3C6 vector on the chromanol group makes with the bilayer normal.

$102 \times 127 \mathrm{~mm}(300 \times 300$ DPI $)$ 